\title{
Engineered PQQ-glucose dehydrogenase as a universal biosensor platform
}

Zhong Guo ${ }^{1}$, Lindy Murphy ${ }^{2}$, Viktor Stein ${ }^{1}$, Wayne J ohnston ${ }^{1}$, Siro Alcala Perez ${ }^{2}$ and Kirill Alexandrov ${ }^{1 *}$

\section{Supplementary information:}

Materials and Methods

\section{Chimeric gene construction and protein expression and purification}

The chimeric GDH genes were constructed by the Gibson Assembly method according to the manufacturer's instructions (New England Biolabs) and cloned into pET28a vector. The full list of sequences are listed. The gene fragments were either generated by PCR or purchased as synthetic gBlocks from Integrated DNA Technologies. Expression and purification of Acinetobacter calcoaceticus GDH or its variants was performed as described ${ }^{[1]}$. The proteins of cyclosporine sensor were purified as described previously ${ }^{[2]}$ with minor modifications: Following Ni-NTA purification the pooled enzyme-containing fractions were supplemented with EDTA to the final concentration $5 \mathrm{mM}$ and dialyzed against buffer containing $20 \mathrm{mM}$ $\mathrm{KH}_{2} \mathrm{PO}_{4} \mathrm{pH} 7.0$ and 5mM EDTA for 10 hours. Subsequently EDTA was removed by dialyzing the sample against the buffer containing $20 \mathrm{mM} \mathrm{KH}_{2} \mathrm{PO}_{4} \mathrm{pH} 7.0$ only. Purified $\mathrm{GDH}$ was reconstituted with its co-factor $\mathrm{PQQ}$ using a 1:1.5 molar ratio.

\section{Spectrophotometric analysis of GDH enzymatic activity}

The GDH enzyme assay was performed as described ${ }^{[3]}$. Briefly, the $1.5 \mathrm{~mL}$ reaction volume comprised $20 \mathrm{mM}$ glucose, $0.6 \mathrm{mM}$ phenazine methosulfate (PMS), $0.06 \mathrm{mM}$ 2,6dichlorophenolindophenol (DCPIP), $10 \mathrm{mM}$ MOPS ( $\mathrm{pH}$ 7.0), $50 \mu \mathrm{M} \mathrm{MgCl}$ and the chosen concentration of the enzyme. The enzymatic assay was performed at $25{ }^{\circ} \mathrm{C}$ by monitoring the decrease in absorbance of DCPIP at $600 \mathrm{~nm}$.

\section{Data fitting}

As rapamycin binds to FKBP with $K_{d}$ close to $0.2 \mathrm{nM}$ we treated FKBP12:rapamycin binary complex as one component in our analysis. The maximal reaction rate was determined as $\mathrm{K}_{\mathrm{obs}}$ when GDH-FKBP was fully activated by $\mathrm{FRB}-\mathrm{GDH}_{1-153}$ in the presence of rapamycin. We 
assumed that the observed rate was in a linear relationship with the ternary complex formation. The following equations were used for data fitting:

$$
\begin{gathered}
K_{d}=\frac{[F R B][F K B P \bullet \text { Rapamycin }]}{[F R B \bullet F K B P \bullet \text { Rapamycin }]} \\
C_{F K B P}=[F K B P \bullet \text { Rapamycin }]+[F R B \bullet F K B P \bullet \text { Rapamycin }] \\
C_{F R B}=[F R B]+[F R B \bullet F K B P \bullet \text { Rapamycin }] \\
k_{\text {obs }}=k_{\text {max }} \bullet \frac{[F R B \bullet F K B P \bullet \text { Rapamycin }]}{C_{F K B P}}
\end{gathered}
$$

$\mathrm{K}_{\mathrm{d}}$ : dissociation constant between GDH-FKBP: rapamycin and FRB-GDH ${ }_{1-153}$ [FRB]: free concentration of FRB-GDH ${ }_{1-153}$

[FKBP:rapamycin]: free concentration of GDH-FKBP: rapamycin binary complex [FRB: FKBP:rapamycin]: concentration of FRB-GDH ${ }_{1-153}$ :GDH-FKBP: rapamycin ternary complex

$\mathrm{C}_{\mathrm{FKBP}}$ : initial concentration of GDH-FKBP

$\mathrm{C}_{\mathrm{FRB}}$ : initial concentration of FRB-GDH $\mathrm{H}_{1-153}$

$\mathrm{k}_{\max }$ : maximal rate, when GDH-FKBP was fully activated by FRB-GDH ${ }_{1-153}$ in the presence of rapamycin

$k_{\mathrm{obs}}$ The fit of the initial reaction phase

\section{Electrochemical analysis of $\alpha$-Amylase biosensor}

Chronoamperometric measurements were carried out using an Autolab PGSTAT204 potentiostat (Eco Chemie) interfaced to DropSens disposable screen printed gold electrodes (Cat\#DRP-C220BT). A fresh sensor was used for each measurement. Reactions contained: 22.5nM N-terminal component of amylase biosensor, $19 \mathrm{nM}$ of C-terminal component of amylase biosensor PQQ, 3.7nM TVMV, 1.0mM 1-methoxy-5-methylphenazinium methyl sulfate, $50 \mathrm{mM}$ glucose, $2.0 \mathrm{mM} \mathrm{MgCl} 2,50 \mathrm{uM} \mathrm{CaCl}$ and 0 to $1000 \mathrm{nM}$ human salivary $\alpha-$ amylase in $45 \mu \mathrm{l}$ total volume at pH 7.4 PBS. Reactions were started with the addition of 1mM 1-methoxy-5-methylphenazinium methyl sulfate and $50 \mathrm{mM}$ glucose and incubated at room temperature for 30 seconds before being pipetted onto the electrode surface. After a 
delay time of 180 seconds, chronoamperometry was carried out for $5 \mathrm{~s}$ at $+0.4 \mathrm{~V}$ versus the imbedded silver strip on the screen printed electrode, with data generally reported as current at the $5 \mathrm{~s}$ time point versus amylase concentration.

\section{References:}

[1] A. J. Olsthoorn, J. A. Duine, Arch. Biochem. Biophys. 1996, 336, 42-8.

[2] L. Jin, S. C. Harrison, Proc. Natl. Acad. Sci. 2002, 99, 13522-13526.

[3] Z Guo, W. A. Johnston, V. Stein, P. Kalimuthu, S. Perez-Alcala, P. V Bernhardt, K. Alexandrov, Chem. Commun. (Camb). 2016, 52, 485-8.

Table S1: Protein components of developed biosensors

\begin{tabular}{|c|c|c|}
\hline Rapamycin & $\mathrm{GDH}_{1-153}-\mathrm{FRB}-\mathrm{His}_{6}$ & $\mathrm{GDH}_{1-153 \text { inactive mutant }}-\mathrm{FKBP}-\mathrm{GDH}_{155-454}-\mathrm{His}_{6}$ \\
\hline FK506 & $\begin{array}{l}\text { Co-expression of } \\
\mathrm{GDH}_{1-153} \text {-Calcinurin } \\
\mathrm{His}_{6} \\
\mathrm{His}_{6} \text {-Calcinurin A }\end{array}$ & $\mathrm{GDH}_{1-153 \text { inactive mutant }}-\mathrm{FKBP}-\mathrm{GDH}_{155-454}-\mathrm{His}_{6}$ \\
\hline Cyclosporine A & $\begin{array}{l}\text { Co-expression of } \\
\mathrm{GDH}_{1-153} \text {-Calcinurin } \\
\mathrm{His}_{6} \\
\mathrm{His}_{6} \text {-Calcinurin A }\end{array}$ & $\mathrm{GDH}_{1-153 \text { inactive mutant }}-\mathrm{GDH}_{155-454}-\mathrm{CYPA}-\mathrm{His}_{6}$ \\
\hline $\begin{array}{l}\text { Salivary alpha } \\
\text { amylase }\end{array}$ & $\mathrm{GDH}_{1-153}-\mathrm{VHH}_{1 \mathrm{~K} \times \mathrm{V}}-\mathrm{His}_{6}$ & $\begin{array}{l}\mathrm{GDH}_{1-153} \text { inactive mutant }-\mathrm{VHH}_{1 \mathrm{BVN}}-\mathrm{GDH}_{155-454^{-}} \\
\mathrm{HiS}_{6}\end{array}$ \\
\hline $\begin{array}{l}\text { Thrombin } \\
\text { sensor }\end{array}$ & $\begin{array}{l}\mathrm{GDH}_{1-153}-\mathrm{SH} 3-\mathrm{Thr}-\mathrm{SH} 3 \\
\text { binding peptide-His } 6\end{array}$ & $\begin{array}{l}\mathrm{GDH}_{1-153} \text { inactive } \text { mutant}^{-} \text {Thr-SH3 binding } \\
\text { peptide-GDH }{ }_{155-454}-\mathrm{His}_{6}\end{array}$ \\
\hline $\begin{array}{l}\text { Factor Xa } \\
\text { sensor }\end{array}$ & $\begin{array}{l}\mathrm{GDH}_{1-153}-\mathrm{SH} 3-\mathrm{FX}-\mathrm{SH} 3 \\
\text { binding peptide-His } \\
6\end{array}$ & $\begin{array}{l}\mathrm{GDH}_{1-153} \text { inactive mutant }-\mathrm{FX}-\mathrm{SH} 3 \text { binding } \\
\text { peptide-GDH } \\
155-454-\mathrm{HiS}_{6}\end{array}$ \\
\hline
\end{tabular}

Sequences of biosensors used in this study:

Rapamycin sensor: 


\section{GDH fragments are underlined}

Component1: GDH(1-153AA)-FRB

DVPLIPSQFAKAKSENFDKKVILSNLNKPHALLWGPDNQIWLTERATGKILRVN PESGSVKTVFQVPEIVNDADGQNGLLGFAFHPDFKNNPYIYISGTFKNPKSTDK ELPNQTIIRRYTYNKSTDTLEKPVDLLAGLPSSKDHQSGRLVIGPGGSGSGSGGL WHEMWHEGLEEASRLYFGERNVKGMFEVLEPLHAMMERGPQTLKETSFNQA YGRDLMEAQEWCRKYMKSGNVKDLTQAWDLYYHVFRRISGKLAAALEHHHHH $\mathrm{H}$

Component2: GDH(1-153AA, Q76A, D143A,H144A)-TVMV cleavage site-FKBP-GDH(155-454AA)

DVPLIPSQFAKAKSENFDKKVILSNLNKPHALLWGPDNQIWLTERATGKILRVN PESGSVKTVFQVPEIVNDADGANGLLGFAFHPDFKNNPYIYISGTFKNPKSTDK ELPNQTIIRRYTYNKSTDTLEKPVDLLAGLPSSKAAQSGRLVIGPGGSGGETVRF QSGGSGSGGVQVETISPGDGRTFPKRGQTCVVHYTG FKF $\underline{M} L G K Q E V I R G W E E G V A Q \underline{M} S V G Q R A K L T I S P D Y A Y G A T G H P G I I P P H A T L V$ FDVELLKLEGSGQKIYYTIGDQGRNQLAYLFLPNQAQHTPTQQELNGKDYHTY MGKVLRLNLDGSIPKDNPSFNGVVSHIYTLGHRNPQGLAFTPNGKLLQSEQGP NSDDEINLIVKGGNYGWPNVAGYKDDSGYAYANYSAAANKTIKDLAQNGVKV AAGVPVTKESEWTGKNFVPPLKTLYTVQDTYNYNDPTCGEMTYICWPTVAPSS AYVYKGGKKAITGWENTLLVPSLKRGVIFRIKLDPTYSTTYDDAVPMFKSNNRY RDVIASPDGNVLYVLTDTAGNVQKDDGSVTNTLENPGSLIKFTYKAKHHHHHH

\section{FK506 sensor:}

Component1-1: GDH(1-153AA)-Calcineurin alpha subunit

DVPLIPSQFAKAKSENFDKKVILSNLNKPHALLWGPDNQIWLTERATGKILRVN PESGSVKTVFQVPEIVNDADGQNGLLGFAFHPDFKNNPYIYISGTFKNPKSTDK ELPNQTIIRRYTYNKSTDTLEKPVDLLAGLPSSKDHQSGRLVIGPGGSGGSGGSG SGGSGGGNEASYPLEMCSHFDADEIKRLGKRFKKLDLDNSGSLSVEEFMSLPEL QQNPLVQRVIDIFDTDGNGEVDFKEFIEGVSQFSVKGDKEQKLRFAFRIYDMDK DGYISNGELFQVLKMMVGNNLKDTQLQQIVDKTIINADKDGDGRISFEEFCAV $V G G L D I H K K M V V D V K L A A A L H H H H H H$

Component1-2: Calcineurin beta subunit

AHHHHHHSSGTSEPKAIDPKLSTTDRVVKAVPFPPSHRLTAKEVFDNDGKPRV DILKAHLMKEGRLEESVALRIITEGASILRQEKNLLDIDAPVTVCGDIHGQFFDL MKLFEVGGSPANTRYLFLGDYVDRGYFSIECVLYLWALKILYPKTLFLLRGNHEC RHLTEYFTFKQECKIKYSERVYDAC M DAFCLPLAAL MNQQFLCVHGGLSPEIN TLDDIRKLDRFKEPPAYGP MCDILWSDPLEDFGNEKTQEHFTHNTVRGCSYFYS YPAVCEFLQHNNLLSILRAHEAQDAGYR MYRKSQTTGFPSLITIFSAPNYLDVY

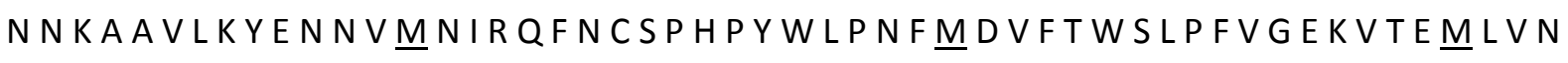
VLNICSDDELGSEEDGFDGATAAARLVTAGLVLA 
Component 3: GDH(1-153AA, Q76A, D143A,H144A)-TVMV cleavage site-FKBP-GDH(155-454AA)

DVPLIPSQFAKAKSENFDKKVILSNLNKPHALLWGPDNQIWLTERATGKILRVN PESGSVKTVFQVPEIVNDADGANGLLGFAFHPDFKNNPYIYISGTFKNPKSTDK ELPNQTIIRRYTYNKSTDTLEKPVDLLAGLPSSKAAQSGRLVIGPGGSGGETVRF QSGGSGSGGVQVETISPGDGRTFPKRGQTCVVHYTG FKF $\underline{M} L G K Q E V I R G W E E G V A Q \underline{M} S V G Q R A K L T I S P D Y A Y G A T G H P G I I P P H A T L V$ FDVELLKLEGSGQKIYYTIGDQGRNQLAYLFLPNQAQHTPTQQELNGKDYHTY MGKVLRLNLDGSIPKDNPSFNGVVSHIYTLGHRNPQGLAFTPNGKLLQSEQGP NSDDEINLIVKGGNYGWPNVAGYKDDSGYAYANYSAAANKTIKDLAQNGVKV AAGVPVTKESEWTGKNFVPPLKTLYTVQDTYNYNDPTCGEMTYICWPTVAPSS AYVYKGGKKAITGWENTLLVPSLKRGVIFRIKLDPTYSTTYDDAVPMFKSNNRY RDVIASPDGNVLYVLTDTAGNVQKDDGSVTNTLENPGSLIKFTYKAKHHHHHH

\section{Cyclosporin A sensor:}

Component1-1: GDH(1-153AA)-Calcineurin alpha subunit

DVPLIPSQFAKAKSENFDKKVILSNLNKPHALLWGPDNQIWLTERATGKILRVN PESGSVKTVFQVPEIVNDADGQNGLLGFAFHPDFKNNPYIYISGTFKNPKSTDK ELPNQTIIRRYTYNKSTDTLEKPVDLLAGLPSSKDHQSGRLVIGPGGSGGSGGSG SGGSGGGNEASYPLEM CSHFDADEIKRLGKRFKKLDLDNSGSLSVEEFMSLPEL QQNPLVQRVIDIFDTDGNGEVDFKEFIEGVSQFSVKGDKEQKLRFAFRIYDMDK DGYISNGELFQVLK $\underline{M} \underline{M} V G N N L K D T Q L Q Q I V D K T I I N A D K D G D G R I S F E E F C A V$ $V G G L D I H K K \underline{M} V V D V K L A A A L E H H H H H H$

Component1-2: Calcineurin beta subunit

AHHHHHHSSGTSEPKAIDPKLSTTDRVVKAVPFPPSHRLTAKEVFDNDGKPRV DILKAHLMKEGRLEESVALRIITEGASILRQEKNLLDIDAPVTVCGDIHGQFFDL MKLFEVGGSPANTRYLFLGDYVDRGYFSIECVLYLWALKILYPKTLFLLRGNHEC RHLTEYFTFKQECKIKYSERVYDAC M DAFDCLPLAAL TLDDIRKLDRFKEPPAYGP MCDILWSDPLEDFGNEKTQEHFTHNTVRGCSYFYS YPAVCEFLQHNNLLSILRAHEAQDAGYRMYRKSQTTGFPSLITIFSAPNYLDVY NNKAAVLKYENNVMIRQFNCSPHPYWLPNFM DVFTWSLPFVGEKVTEMLVN VLNICSDDELGSEEDGFDGATAAARLVTAGLVLA

Component2: GDH(1-153AA, Q76A, D143A,H144A)-TVMV cleavage site-GDH(155-454AA)-

Cyclophilin

DVPLIPSQFAKAKSENFDKKVILSNLNKPHALLWGPDNQIWLTERATGKILRVN PESGSVKTVFQVPEIVNDADGANGLLGFAFHPDFKNNPYIYISGTFKNPKSTDK ELPNQTIIRRYTYNKSTDTLEKPVDLLAGLPSSKAAQSGRLVIGPGSGETVRFQS GSGQKIYYTIGDQGRNQLAYLFLPNQAQHTPTQQELNGKDYHTYMGKVLRLNL 
DGSIPKDNPSFNGVVSHIYTLGHRNPQGLAFTPNGKLLQSEQGPNSDDEINLIV KGGNYGWPNVAGYKDDSGYAYANYSAAANKTIKDLAQNGVKVAAGVPVTKES EWTGKNFVPPLKTLYTVQDTYNYNDPTCGEMTYICWPTVAPSSAYVYKGGKKA ITGWENTLLVPSLKRGVIFRIKLDPTYSTTYDDAVPMFKSNNRYRDVIASPDGN VLYVLTDTAGNVQKDDGSVTNTLENPGSLIKFTYKAKGGSGGSGGSGGSGGSG GMVNPTVFFDIAVDGEPLGRVSFELFADKVPKTAENFRALSTGEKGFGYKGSCF HRIIPGFMCQGGDFTRHNGTGGKSIYGEKFEDENFILKHTGPGILSMANAGPNT NGSQFFICTAKTEWLDGKHVVFGKVKEGMNIVEAMERFGSRNGKTSKKITIAD $C G Q L E K L A A A L E H H H H H$

\section{Thrombin sensor:}

Component1: GDH(1-153AA)-SH3-Thrombin site-SH3L Green:SH3

Yellow: Thrombin cleavage site

Red: SH3 binding ligand

DVPLIPSQFAKAKSENFDKKVILSNLNKPHALLWGPDNQIWLTERATGKILRVN PESGSVKTVFQVPEIVNDADGQNGLLGFAFHPDFKNNPYIYISGTFKNPKSTDK ELPNQTIIRRYTYNKSTDTLEKPVDLLAGLPSSKDHQSGRLVIGP

GGSGGSGGA E Y VRALFDFN GNDEEDLPFKK G DILRIR DKPEE QWW N A D SE GKR G MI I VPY V K Y YGSGGSGG LVPRG VGGSGGSG PPP PLPPKRRRGG KLAAALEHHHHH $\mathrm{H}$

Component2: GDH(1-153AA, Q76A, D143A,H144A)-Thrombin site-SH3L-GDH(155-454AA) Yellow: Thrombin cleavage site, Red: SH3 binding ligand

DVPLIPSQFAKAKSENFDKKVILSNLNKPHALLWGPDNQIWLTERATGKILRVN PESGSVKTVFQVPEIVNDADGANGLLGFAFHPDFKNNPYIYISGTFKNPKSTDK ELPNQTIIRRYTYNKSTDTLEKPVDLLAGLPSSKAAQSGRLVIGP

G GLVPRGVGG PPPPLPPKRRRGGSGGSGGQKIYYTIGDQGRNQLAYLFLPNQAQH TPTQQELNGKDYHTY M GKVLRLNLDGSIPKDNPSFNGVVSHIYTLGHRNPQGL AFTPNGKLLQSEQGPNSDDEINLIVKGGNYGWPNVAGYKDDSGYAYANYSAAA NKTIKDLAQNGVKVAAGVPVTKESEWTGKNFVPPLKTLYTVQDTYNYNDPTCG EMTYICWPTVAPSSAYVYKGGKKAITGWENTLLVPSLKRGVIFRIKLDPTYSTTY DDAVP M FKSNNRYRDVIASPDGNVLYVLTDTAGNVQKDDGSVTNTLENPGSLI KFTYKAKHHHHHH

\section{Factor Xa sensor with one cleavage site:}

Component1: GDH(1-153AA)-SH3-Factor Xa site-SH3L, Green: SH3, Yellow "IEGR": Xa cleavage site 
Yellow "PPPPLPPKRRR": SH3 binding ligand

DVPLIPSQFAKAKSENFDKKVILSNLNKPHALLWGPDNQIWLTERATGKILRVN PESGSVKTVFQVPEIVNDADGQNGLLGFAFHPDFKNNPYIYISGTFKNPKSTDK ELPNQTIIRRYTYNKSTDTLEKPVDLLAGLPSSKDHQSGRLVIGP

G GSGGSGGAEYVRALFDFNGNDEEDLPFKKGDILRIRDKPEEQWWNAEDSEGK

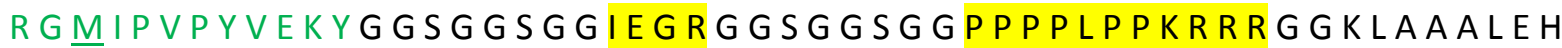
$\mathrm{HHHHH}$

Component2: GDH(1-153AA, Q76A, D143A,H144A)-one Factor Xa site-SH3L-GDH(155-454AA), Yellow "IEGR": Xa cleavage site, Yellow “PPPPLPPKRRR": SH3 binding ligand

DVPLIPSQFAKAKSENFDKKVILSNLNKPHALLWGPDNQIWLTERATGKILRVN PESGSVKTVFQVPEIVNDADGANGLLGFAFHPDFKNNPYIYISGTFKNPKSTDK ELPNQTIIRRYTYNKSTDTLEKPVDLLAGLPSSKAAQSGRLVIGP

GGIEGRGGSGPPPPLPPKRRRGGSGGSGGQKIYYTIGDQGRNQLAYLFLPNQA QHTPTQQELNGKDYHTY $\underline{M} G K V L R L N L D G S I P K D N P S F N G V V S H I Y T L G H R N P Q$ GLAFTPNGKLLQSEQGPNSDDEINLIVKGGNYGWPNVAGYKDDSGYAYANYSA A A NKTIKDLAQNGVKVAAGVPVTKESEWTGKNFVPPLKTLYTVQDTYNYNDPT CGEMTYICWPTVAPSSAYVYKGGKKAITGWENTLLVPSLKRGVIFRIKLDPTYST TYDDAVP M FKSNNRYRDVIASPDGNVLYVLTDTAGNVQKDDGSVTNTLENPGS LIKFTYKAKHHHHHH

(SEQ ID NO:47)

\section{Factor Xa sensor with three cleavage site:}

Component1: GDH(1-153AA)-SH3-three Factor Xa site-SH3L, Yellow "IEGR" “IDGR": Xa cleavage site Yellow "PPPPLPPKRRR": SH3 binding ligand

DVPLIPSQFAKAKSENFDKKVILSNLNKPHALLWGPDNQIWLTERATGKILRVN PESGSVKTVFQVPEIVNDADGQNGLLGFAFHPDFKNNPYIYISGTFKNPKSTDK ELPNQTIIRRYTYNKSTDTLEKPVDLLAGLPSSKDHQSGRLVIGP

GGSGGSGGAEYVRALFDFNGNDEEDLPFKKGDILRIRDKPEEQWWNAEDSEGK RG $\underline{M}$ IPVPYVEKYGGSGGSGGIEGRGIDGRGIEGRGGSGGSGGPPPPLPPKRRR GGKLAAALEHHHHHH

Component2: GDH(1-153AA, Q76A, D143A,H144A)-three Factor Xa site-SH3L-GDH(155-454AA), Yellow "IEGR" "IDGR": Xa cleavage site, Yellow "PPPPLPPKRRR": SH3 binding ligand 
DVPLIPSQFAKAKSENFDKKVILSNLNKPHALLWGPDNQIWLTERATGKILRVN PESGSVKTVFQVPEIVNDADGANGLLGFAFHPDFKNNPYIYISGTFKNPKSTDK ELPNQTIIRRYTYNKSTDTLEKPVDLLAGLPSSKAAQSGRLVIGP

G GIEGRGIDGR GIEGRGGSGPPPPLPPKRRRGGSGGSGG

QKIYYTIGDQGRNQLAYLFLPNQAQHTPTQQELNGKDYHTYM GKVLRLNLDGS IPKDNPSFNGVVSHIYTLGHRNPQGLAFTPNGKLLQSEQGPNSDDEINLIVKGG NYGWPNVAGYKDDSGYAYANYSAAANKTIKDLAQNGVKVAAGVPVTKESEWT GKNFVPPLKTLYTVQDTYNYNDPTCGEMTYICWPTVAPSSAYVYKGGKKAITG WENTLLVPSLKRGVIFRIKLDPTYSTTYDDAVPMFKSNNRYRDVIASPDGNVLY VLTDTAGNVQKDDGSVTNTLENPGSLIKFTYKAKHHHHHH

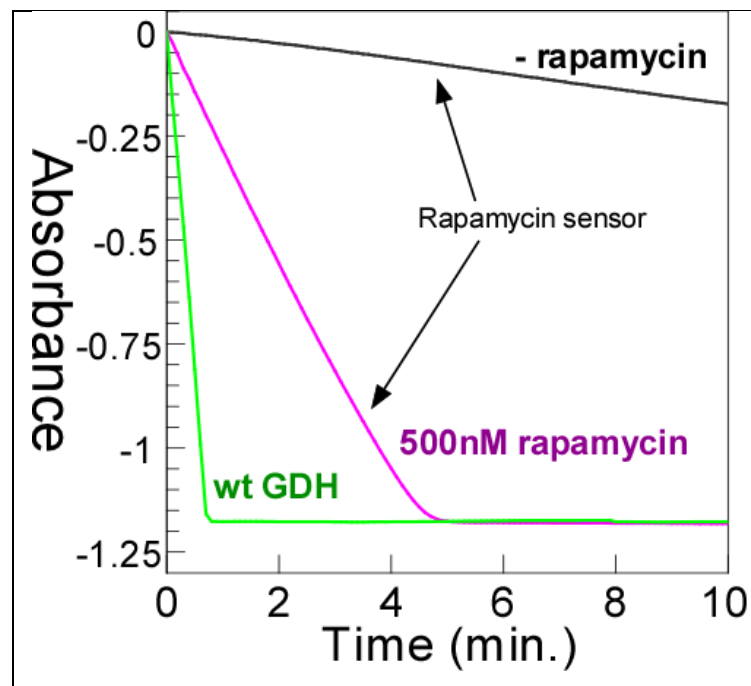

Figure S1 Enzymatic activity of GDH-based rapamycin two component biosensor in comparison with wt GDH. The activity of the split enzyme was monitored by change of $600 \mathrm{~nm}$ absorption of $60 \mu \mathrm{M}$ electron accepting dye dichlorophenolindophenol in the presence of $0.6 \mathrm{mM}$ electron mediator phenazine methosulphate, $20 \mathrm{mM}$ of glucose, $50 \mu \mathrm{M} \mathrm{CaCl}_{2}, 15 \mathrm{nM}$ of FRB-GDH ${ }_{1-153}, 10 \mathrm{nM}$ of inactive full length of GDH-FKBP12 and 500nM rapamycin. In the assay with wt $\mathrm{GDH}, 10 \mathrm{nM}$ of the enzyme were used. The initial linear phase of the reaction was fitted to a linear function to obtain $\mathrm{K}_{\mathrm{obs}}$ of 1.6 for the wt GDH, 0.3 for the fully activated rapamycin biosensor and 0.016 for rapamycin biosensor in the absence of rapamycin. 

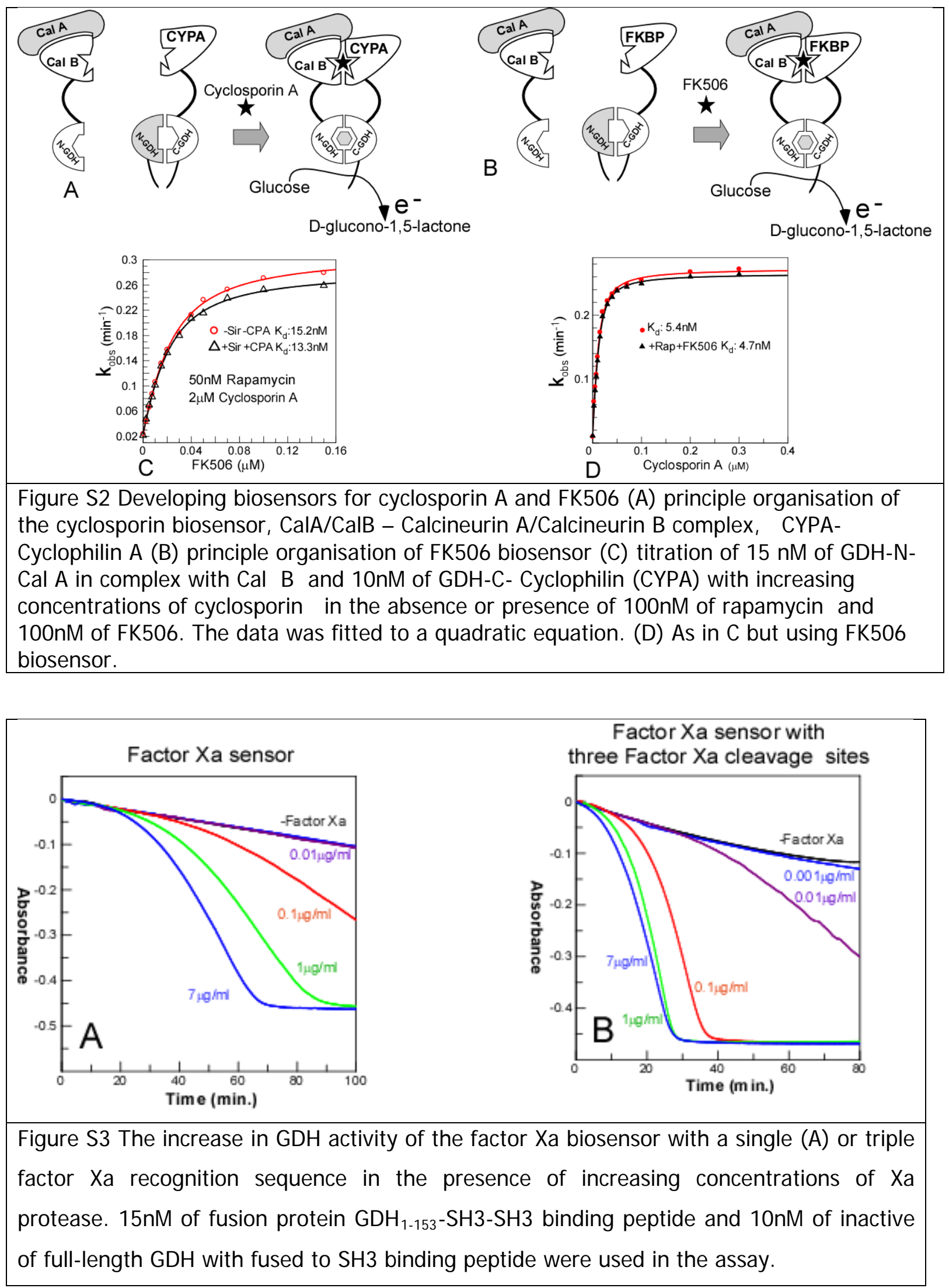


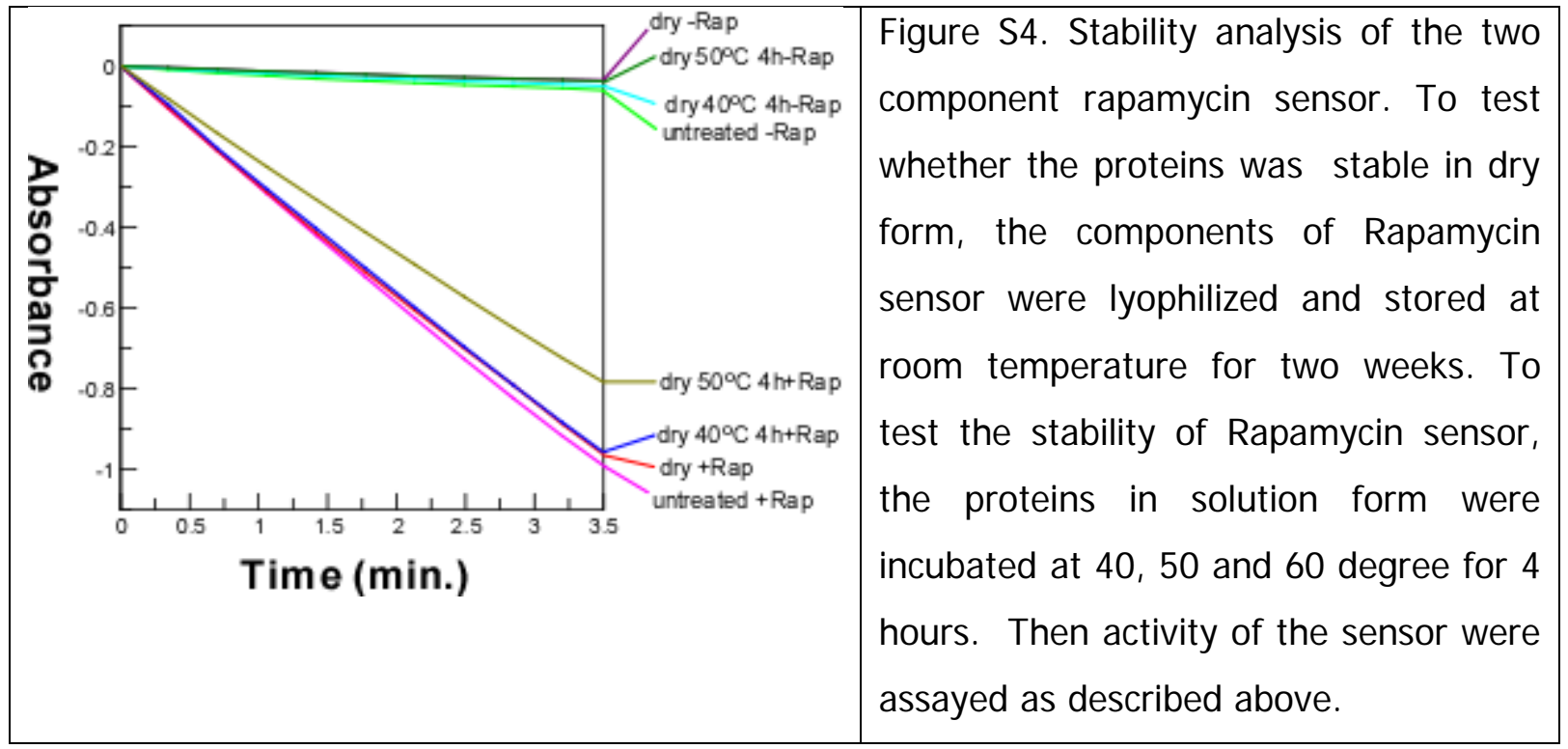

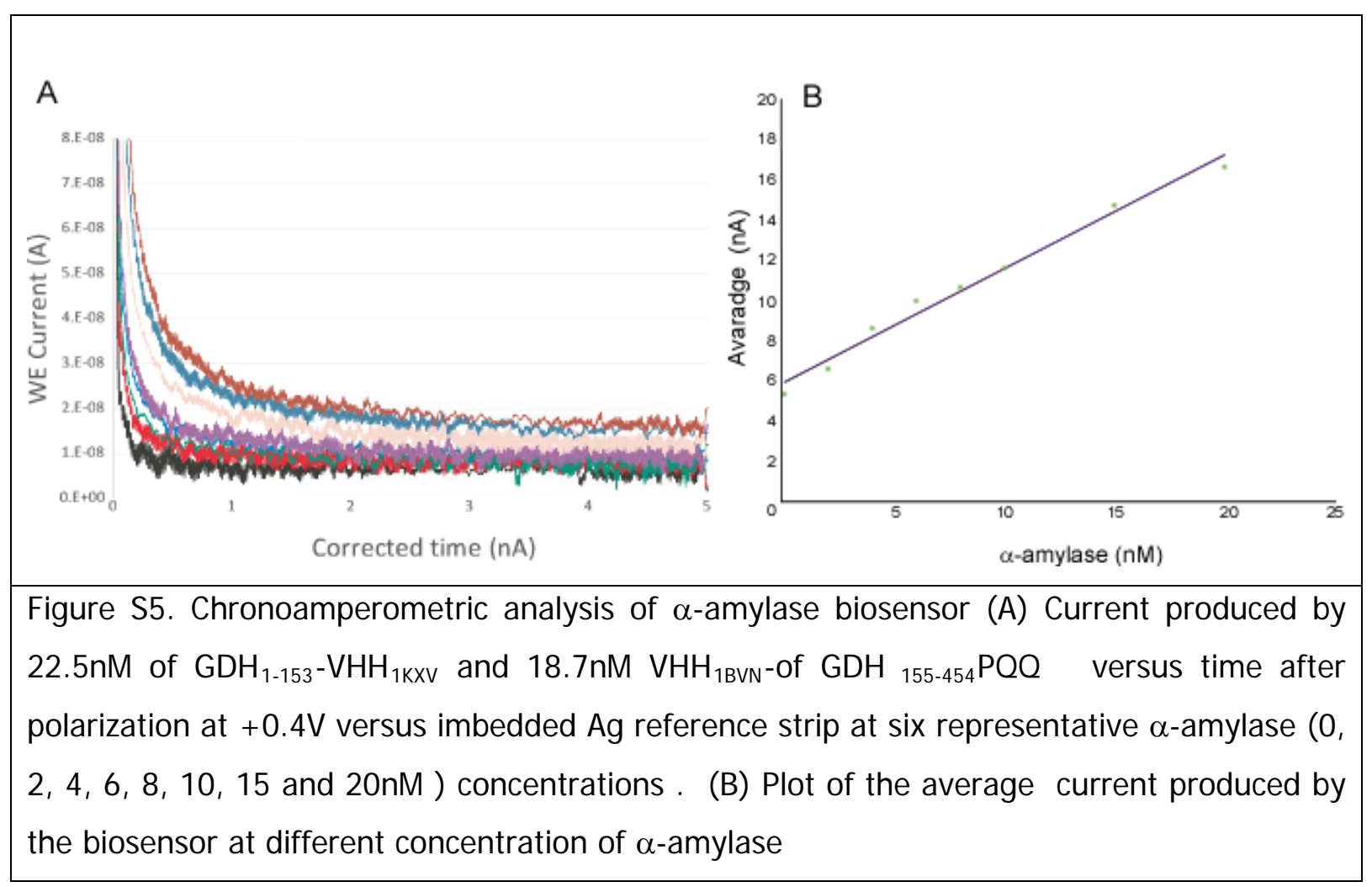

